\title{
La gestión de bibliotecas en España (2005- 2010): tendencias y cambios significativos
}

\author{
Ana ReYes Pacios LozANO \\ Universidad Carlos III de Madrid. Departamento de Biblioteconomía y Documentación \\ areyes@bib.uc3m.es
}

Recibido: junio 2011

Aceptado: octubre 2011

Resumen: Se ofrece una perspectiva global de las tendencias relacionadas con la gestión de bibliotecas en España durante los últimos cinco años. Los temas identificados de mayor protagonismo tienen su repercusión en la aplicación de técnicas de gestión y también en la gestión de los recursos de las bibliotecas (colección, recursos humanos, espacios y equipamiento), lo que permite identificar algunos cambios significativos. La revisión y análisis de los trabajos publicados en este período en revistas, actas de congresos, seminarios y jornadas, fundamentalmente, además de la consideración de los grupos de trabajo creados en el seno de distintas asociaciones profesionales, proporciona las líneas de interés fundamentales. En ellas se han centrado los profesionales y docentes dedicados a la gestión a lo largo de este período aportando experiencias, reflexiones y soluciones.

Palabras clave: Tendencias bibliotecas; cambios gestión de bibliotecas; técnicas de gestión; gestión de recursos; Marketing; Planificación; Evaluación; Calidad; Gestión del conocimiento; Gestión de la colección; Responsabilidad Social Corporativa.

\section{Library management in Spain (2005-2010): trends and significant changes}

\begin{abstract}
An overview of the management trends in relation to Spanish libraries during the last five years is offered. The most significant issues identified have repercussions on the implementation of management techniques and on the management of library resources (collection, human resources, spaces, and equipment), showing some significant changes. Examination and analysis of the works published during that period in journals, and of proceedings of congresses, conferences and seminars, essentially, as well as the developments of working groups created within different professional associations, unveil the fundamental lines of interest. These are the lines on which professionals and teachers devoted to management have focused over that period, contributing experiences, reflections and solutions.
\end{abstract}

Keywords: Library trends; Library management changes; Management techniques; Resource Management; Marketing, Planing, Evaluation, Quality, Knowledge Management; Management collection; Corporate Social Responsability. 


\section{INTRODUCCIÓN}

Los trabajos relacionados con la gestión de las bibliotecas son el reflejo que lo que hacen los profesionales que tienen que ver con su gestión para adaptarse continuamente al entorno turbulento en el que operan. Las tendencias económicas, sociales, políticas y de cualquier otra índole repercuten en la gestión. Más aún cuando las bibliotecas no son consideradas una prioridad en la institución y tienen que acostumbrarse a convivir con las dificultades, incluso a gestionar con eficacia los cambios para demostrar que son capaces de cumplir con sus funciones a pesar de la adversidad.

El objetivo de este trabajo es identificar los temas que han suscitado mayor interés en España a lo largo de los últimos cinco años y que han repercutido en la gestión de las bibliotecas. La mayoría han sido expuestos en los trabajos realizados por los profesionales que allí trabajan, muchas veces como resultado de aplicaciones prácticas en sus centros de trabajo o reflexiones ante determinadas situaciones. También por los docentes del área de Biblioteconomía y la Documentación, como resultado de sus investigaciones. No todos los temas identificados afectan por igual a todas las bibliotecas, presentando un grado de desarrollo diferente según el tipo de biblioteca de la que se trate. Incluso algunos como, por ejemplo, la puesta en marcha del Espacio Europeo de Educación Superior, ha afectado exclusivamente a las universitarias.

\section{MÉTODO}

El punto de partida para la revisión de este período han sido las distintas búsquedas realizadas en la base de datos de Biblioteconomía y Documentación del Consejo Superior de Investigaciones Científicas. La búsqueda y localización de la mayoría de los trabajos más recientes se hizo mediante el Google académico.

Para identificar tendencias se consideró imprescindible contar con las presentaciones y actas de reuniones de distinto tipo (congresos, seminarios, jornadas) que se convocan en el marco de consorcios, redes o grupos de usuarios. Algunas, ya tradicionales, y con peso a nivel nacional reúnen tanto a profesionales como a docentes que se centran en aquellos temas que son objeto de atención preferente en un momento determinado. Es el caso, por ejemplo, de las Jornadas de la Federación Española de las sociedades de Archivística, Biblioteconomía y Documentación (FESABID), celebradas cada dos años, las Jornadas de Gestión de la información, de carácter anual y organizadas por la Sociedad Española de Información y Documentación Científica (SEDIC), los Congresos Nacionales de Bibliotecas Públicas, celebrados cada dos años en el que se dan cita un gran número de profesionales procedentes de todas las comunidades autónomas y dan una muestra actualizada de la situación 
bibliotecaria en España y las diversas Jornadas sectoriales organizadas por la Red de Bibliotecas Universitarias (REBIUN) en las que se analizan los temas que preocupan en el ámbito de las bibliotecas universitarias. Estos y otros eventos periódicos de este tipo, celebrados en nuestro país, proporcionan una buena radiografía del avance real de los trabajos y proyectos que se llevan a cabo en las bibliotecas, entre otros servicios de información.

Como complemento, se ha contado con los grupos de trabajo formados en las principales asociaciones profesionales y redes (SEDIC, REBIUN, FESABID), como elemento representativo de una línea de interés o tema que preocupa entre la profesión, sobre la que se quiere trabajar y a la que se pretende dar respuesta de forma consensuada.

Aunque el trabajo se centra en los últimos cinco años, en algún caso, dado el comienzo y la continuación lógica de estos temas antes y después del período fijado, se ha citado algún trabajo significativo inmediatamente anterior o posterior al ámbito temporal establecido.

Los trabajos en los que se ha basado este artículo han sido, fundamentalmente, artículos de revistas y actas de diferentes eventos. No obstante, en algunos temas, al contar con monografías o documentos de trabajo significativos, se han incluido también este tipo de publicaciones. Dada la gran cantidad de trabajos relacionados con la temática identificada, en la exposición de las tendencias se citan solo algunos ejemplos representativos.

\section{HECHOS RELEVANTES DEL PERÍODO}

A lo largo de este último quinquenio (2005-2010) se pueden identificar cuatro hechos relacionados con el mundo del libro y las bibliotecas que se convierten en temas de atención preferente y tienen un claro protagonismo en la literatura de estos años. Por otra parte, como se verá más adelante, tendrán además una clara repercusión en la gestión de las bibliotecas. A continuación se exponen por orden cronológico de su aparición:

- La entrada del libro electrónico en las bibliotecas, aunque lenta, en particular en las universitarias a partir del 2005. Se pasa del 55,3\% de bibliotecas con libros electrónicos en 2003 al 72,5\% en el 2005 (Pérez Arranz y Moscoso, 2007). Aunque el crecimiento resulta incontestable, en el 2006 el espacio que ocupaban en el conjunto de la colección no se consideraba suficientemente representativo (Alvite Díez y Rodríguez Bravo, 2006). Aún ahora, en el 2011, se admite la lenta integración y los problemas aún por solventar en las bibliotecas universitarias para su plena incorporación (Alonso Arévalo, Cordón García y Gómez Díaz, 2011 a).

- En el caso de las bibliotecas públicas la incorporación es reciente dado que es el 25 de Noviembre de 2009 cuando se aprueba en el Congreso de los 
Diputados la Proposición 161/001344 no de ley sobre el apoyo al libro electrónico en las bibliotecas públicas, que insta, entre otras cosas, a dotar a las bibliotecas de lectores y soportes que permitan la adecuada lectura de los libros electrónicos. La Dirección General del Libro, Archivos y Bibliotecas del Ministerio de Cultura materializa esta proposición en Enero del 2011 poniendo en marcha un programa que tiene por objetivo la introducción del libro electrónico en las bibliotecas públicas del Estado (Ministerio de Cultura, 2011 b).

- En el auge, además, tienen que ver las iniciativas de apoyo a la digitalización y comercialización del libro electrónico, desde la Administración General de Estado, algunas enmarcadas dentro del Plan Avanza, lanzado en el 2005 como un plan quinquenal de impulso a la Sociedad de la Información y que, desde 2009, se transforma en el Plan Avanza 2, ampliándose hasta 2012. Es el marco en el que se establecen ayudas y subvenciones con el objetivo de integrar herramientas tecnológicas y acercar las actividades empresariales a Internet, dirigidos a todos los sectores (Junquera Temprano, 2010).

- Con todas estas acciones, los datos relativos a 2010 evidencian un notable incremento en la edición de libros electrónicos en España, que alcanza el 45,6\% respecto al año anterior (Ministerio de Cultura, 2011 c). La previsión para este año 2011 es que el avance sea aún más notable ya que se prevé que un gran número de editoriales dispongan de más de la mitad de su catálogo digitalizado. Es de desear que estas previsiones se cumplan y que repercutan en el número de títulos que ofrezcan las bibliotecas si, finalmente, las editoriales aclaran sus modelos de negocio.

- La irrupción de las redes sociales en el año 2006, después de que la web 2.0 hiciera aflorar el rol participativo del usuario con su intervención directa, modifica las relaciones con los usuarios y supone una nueva forma de interacción con ellos. En el 2008 se convirtieron en los sitios más visitados.

- El inicio del proceso de adaptación al Espacio Europeo de Educación Superior que marca el Real Decreto 1393/2007. En el curso 2008 se ponen en marcha los primeros Grados siguiendo el modelo de enseñanza centrada en el aprendizaje conforme a la Declaración de Bolonia de 1999.

- La aparición de la crisis económica en el 2008 conlleva, para la Administración Pública, una reducción de los ingresos del Estado y de la capacidad de las personas para sufragar los servicios. Las bibliotecas, como instituciones dependientes de las administraciones públicas, no son ajenas a esta situación y empiezan a sufrir las consecuencias. 


\section{LA REPERCUSIÓN DE LOS TEMAS DE MAYOR PROTAGONISMO EN LA GESTIÓN DE LAS BIBLIOTECAS}

Los cuatro hechos mencionados anteriormente tienen su repercusión en diferentes aspectos relacionados con la gestión de las bibliotecas, en la gestión de sus recursos o en la forma de aplicar las técnicas de gestión, al contar con nuevas herramientas en las que apoyarse. Los temas que podrían ser considerados "top" y el tipo de cambios que han originado en relación con algún aspecto de la gestión se tratan a continuación:

\subsection{E-BOOKS - CAMBIOS EN LA GESTIÓN DE LA COLECCIÓN}

Las bibliotecas universitarias han sido las primeras en España en adquirir libros electrónicos y también en poner en marcha servicios de consulta y préstamo de lectores. Las bibliotecas públicas se han incorporado más tarde, empezando este año a dar los primeros pasos en el préstamo de e-books. Como ya se mencionó, a comienzos de este año 2011 el Ministerio de Cultura pone en marcha un servicio de préstamo de libros electrónicos en las bibliotecas públicas del Estado para que los ciudadanos puedan tomar en préstamo tanto los dispositivos lectores como los propios contenidos o libros electrónicos. La experiencia se inicia con 15 bibliotecas a las que se las dota de 50 dispositivos, el importe de la inversión asciende a 130.000 euros. Otras bibliotecas públicas, como las de la Obra Social de Caja Madrid o las de la Generalitat de Cataluña, ofrecen este servicio desde el 2010 y su impacto en los usuarios ya está siendo objeto de análisis mediante un proyecto en colaboración con el CSIC $^{1}$. Un caso anterior es la biblioteca de Peñaranda de Bracamonte que, desde 2009, impulsa a través del programa "Territorio e-book", la introducción del libro electrónico en la biblioteca pública.

La llegada de los e-books a las bibliotecas hace que se convierta en un tema de atención preferente en la literatura profesional de este período. Tanto los trabajos referidos a bibliotecas universitarias como a las públicas contienen reflexiones y preocupaciones similares y coincidentes, en muchos casos, a pesar de las diferencias: la forma en la que inciden y modifican los hábitos de los usuarios (Pérez Arranz y Moscoso, 2007), la idoneidad de la inversión (Larrañaga Rubio, 2008), sus peculiaridades y características (Millán, 2008), ventajas y desventajas (García Marco, 2008), los factores clave a tener en cuenta a la hora de adquirir y acceder a los mismos (Martín-González y Pivetta, 2008), las nuevas funciones que tiene que realizar el bibliotecario (Margaix-Arnal, 2008), la situación en nuestro país, su evolución y crecimiento (Cordón García, Alonso Arévalo y Martín

\footnotetext{
${ }^{1}$ E-book y el fomento de la lectura Disponible en:

http://bibliotecas.obrasocialcajamadrid.es/biblioadulto/662_97043.htm?idc $=388680 \& \mathrm{idp}=9705$ 5 (Consulta: 13 junio 2011).
} 
Rodero, 2010), la adaptación de las editoriales al nuevo formato (Costa-Knufinke, 2010), las repercusiones en las políticas de adquisiciones y la diferencia de expectativas entre las bibliotecas y la oferta de editores, así como integración de este nuevo recurso en el catálogo de la biblioteca (Cordón García y Alonso Arévalo, 2010), la oferta en las plataformas de venta y su distribución (Alonso Arévalo, Cordón García y Gómez Díaz, 2011 b), a los que hay que añadir algunas experiencias concretas, como el préstamo (Clavero et al., 2009). La reciente monografía de Cordón García, Gómez Díaz y Alonso Arévalo (2011) recoge todas estas cuestiones relativas al libro electrónico de forma pormenorizada.

El interés que ha suscitado este tema se refleja, además, en la formación de grupos de trabajo que investigan aspectos relacionados con su casuística:

- Grupo de trabajo dedicado al "Libro electrónico", formado en 2009 dentro del Ministerio de Cultura, compuesto por expertos y representantes tanto del sector público como privado (docentes y profesionales), vinculados a la creación, industria del libro, las nuevas tecnologías, la lectura, las bibliotecas y la investigación científica cuyo objetivo es conocer el impacto del libro electrónico en el sector y en los hábitos lectores. En el 2008 ya se había creado el Observatorio de la Lectura y del Libro, adscrito al Ministerio de Cultura, con la finalidad de analizar con carácter permanente la situación del libro, la lectura y las bibliotecas. Su línea prioritaria de actuación está centrada en el estudio del impacto del libro electrónico en el sector y en los hábitos lectores. Con anterioridad a esta iniciativa ya se había puesto en marcha en una de las comunidades autónomas españolas, Extremadura, con similares intenciones.

- Grupo de Trabajo "E-Book y universidad", enmarcado dentro del programa Territorio e-book, formado en el 2010 (Fundación Germán Sánchez Ruipérez, 2010) e integrado por un grupo de expertos cuya pretensión es la de conocer el grado de penetración y aceptación de los nuevos dispositivos en los hábitos de lectura de poblaciones bien diferenciadas, reflexionar sobre las textualidades y los soportes, y proponer recomendaciones para una plena alfabetización digital en el ámbito de la educación superior.

La introducción del libro electrónico en las bibliotecas tiene las siguientes repercusiones y cambios en la gestión de la colección, algunas recogidas en los diferentes trabajos citados:

- Concentración de la gestión, al requerirse que las tareas de selección, negociación y contratación las realice la misma persona mientras que antes, en el caso de la colección en los formatos tradiciones, podían estar distribuidas. Las tareas de negociación y contratación alcanzan un destacado protagonismo.

- La selección se va asemejando en algunos aspectos a la de las revistas electrónicas. Puede hacerse de forma individual, título a título, frente al 
modelo de suscripción a colecciones seleccionadas por el propio editor. De este modo la biblioteca, el bibliotecario, pierde protagonismo al comprar paquetes, lotes, como en el caso de las revistas.

- Las opciones de adquisición también varían al existir, básicamente, dos posibilidades: compra a perpetuidad o suscripción anual. La compra implica una adquisición permanente pero resulta más cara mientras que la licencia de acceso es más barata.

- El sistema de adquisición se modifica al poderse hacer mediante editor o agregador. El editor, en general, ofrece colecciones frente al agregador que ofrece la posibilidad de selección libro a libro, además el editor suele utilizar menos DRM con lo cual el acceso al libro es multiusuario.

- El marketing, antes realizado por el bibliotecario, ahora pasa a ser compartido entre la biblioteca y los propios proveedores que se ofrecen para llevar a cabo campañas de difusión (tanto editores como agregadores). De hecho, el $40 \%$ de las editoriales españolas prevé llevar a cabo acciones de promoción y prospección con su oferta digital, según la Segunda Encuesta sobre el libro digital en España (2010).

- La evaluación de la colección tiene que contar ahora con los datos que proporciona el agregador o editor, por lo tanto no proceden de la biblioteca. También se produce un cambio en la forma de recogida de datos (descargas, búsquedas...) así como en la forma de interpretar la rentabilidad (coste/uso más bajo).

\subsection{LA WEB 2.0 Y LAS REDES SOCIALES - CAMBIOS EN LA FORMA DE HACER MARKETING}

La facilidad que supuso la web 2.0 para que cualquiera pueda ser a la vez consumidor y generador de contenidos va unida a la actitud de los usuarios de estas herramientas. De ahí que en su mayoría tengan un componente de red social al agrupar usuarios con intereses comunes. Con posterioridad, la aceptación de las redes sociales más famosas que operan en España, Facebook y Twitter, supone para las bibliotecas una nueva forma de interacción con los usuarios y la introducción de nuevos servicios. El interés que ha suscitado este tema a partir del 2006 se pone de manifiesto en los diferentes eventos, jornadas y congresos, en los que viene siendo objeto de atención constante.

Ante la llegada de las redes sociales las bibliotecas reaccionan de dos formas distintas: unas las rechazan y otras, al contrario, entran de lleno en ellas pero de modo improvisado, sin ninguna planificación. Esta situación marca el contenido de los trabajos centrados en:

- La experiencia que tienen las bibliotecas después de haber entrado en una red social, los aspectos positivos y negativos (Cabre Serra, 2010). Se recomienda, 
antes de nada, analizar en qué redes debe estar la biblioteca teniendo en cuenta al usuario, lo que se quiere transmitir y concretar el lenguaje y estilo de las comunicaciones.

- Las oportunidades y amenazas para las bibliotecas, cómo se están utilizando y el impacto que tienen (Margaix Arnal, 2007). Se cuestiona si el uso de la web social trae consigo un aumento de la visibilidad de la biblioteca en la comunidad y en la sociedad y, por lo tanto, un aumento del conocimiento y uso de los servicios.

- La necesidad de conocer el éxito, el impacto y la rentabilidad de las redes sociales, utilizando datos que permitan conocer el retorno de esta inversión (González Fernández-Villavicencio, 2009 y 2010).

La repercusión de la web 2.0 y las redes sociales ha dado lugar a los siguientes cambios en la gestión de las bibliotecas:

- Mejoras en el marketing con las considerables ventajas atribuidas a esta nueva forma de ponerlo en práctica: la fidelización del usuario, la actualización continua de los contactos de la biblioteca, la captación de nuevos usuarios entre el público potencial, obtención fácil de contactos profesionales, una mayor optimización de recursos, la ampliación de los canales de comunicación con los usuarios, etc., todo ello con un coste mínimo (García Jiménez, 2010).

- La aparición de una nueva función o, mejor dicho, una nueva adaptación o cambio en el quehacer de los bibliotecarios. Se trata de gestionar las relaciones de la biblioteca con su comunidad en un entorno virtual, cometido que se atribuye al nuevo perfil profesional, el "community manager". Se encargaría de medir el impacto en los diferentes medios y plataformas (González Fernández-Villavicencio, 2011).

- La necesidad de gestionar el riesgo, teniendo en cuenta que la biblioteca 2.0 integra múltiples tecnologías en su oferta (Álvarez Marañón, 2010). De ahí que convenga evaluar el contexto, las expectativas y las medidas de seguridad que se pueden aplicar. Entre los activos amenazados por las redes sociales, según Álvarez Marañón (2010), estarían, entre otros: el robo y el uso de la información privada en contra de su propietario, al igual que la propiedad intelectual, el posible ataque a la integridad física, la erosión de la reputación de la biblioteca y del personal, e incluso, los activos financieros pueden malgastarse por una disminución de la productividad de los empleados, etc.

- El uso de nuevas herramientas de evaluación que permitan conocer la producción, el impacto y la visibilidad, además de la necesidad de mantenerse al día de lo que están haciendo otras bibliotecas mediante el benchmarking (Torres-Salinas, 2010). 


\subsection{LA ADAPTACIÓN AL ESPACIO EUROPEO DE EDUCACIÓN SUPERIOR - REPERCUSIONES EN LA GESTIÓN DE ESPACIOS, GESTIÓN DE LA INFORMACIÓN Y GESTIÓN DE PERSONAL EN LAS BIBLIOTECAS UNIVERSITARIAS}

La puesta en marcha del Espacio Europeo de Educación Superior requiere que las bibliotecas realicen una tarea docente que involucre a profesores y a estudiantes en el conocimiento de sus recursos. Las bibliotecas universitarias empiezan a gestionar el cambio para convertirse en Centros de Recursos para el Aprendizaje y la Investigación (CRAI). Este cambio dará lugar a trabajos que se extienden hasta el momento actual (Casal Reyes, 2011), con la celebración de las XI Jornadas CRAI, en los que se viene analizando:

- Las competencias profesionales que deben adquirir los nuevos graduados (Tejada Artigas et al., 2006; Sunyer, 2006)

- La definición de nuevos perfiles profesionales y las habilidades que deben adquirir los bibliotecarios ante los cambios que impone la educación superior (Marzal García-Quismondo, 2006).

- La organización y gestión del nuevo modelo de biblioteca universitaria CRAI-, de cuya definición se había tratado ya con anterioridad (Balagué, 2003 y Taladriz, 2004) y que continuará siendo objeto de interés a lo largo de este período (Domínguez Aroca, 2008), incluso dando a conocer experiencias concretas (Arquero Avilés et al. 2007).

El interés por poner en marcha los CRAIs lleva a varias iniciativas:

- La formación de un grupo de trabajo en REBIUN

- Una línea de su plan estratégico correspondiente al período 2003-2006 dedicada a impulsar la construcción de este nuevo modelo de biblioteca universitaria.

- La celebración de las Jornadas CRAI desde el año 2003, en las que se han venido abordando los espacios, servicios, los modelos organizativos, las competencias y demás aspectos relacionados con el nuevo modelo de biblioteca.

La adaptación al EEES conlleva cambios en la gestión de los siguientes recursos de la biblioteca universitaria:

- El espacio y equipamiento. El nuevo modelo de biblioteca (CRAI) obliga a gestionar el espacio de modo que se puedan crear áreas de trabajo y de encuentro convenientemente equipadas que conviertan la biblioteca física en un punto neurálgico para el aprendizaje.

- El personal. Dado que los bibliotecarios empiezan a desarrollar nuevas funciones resulta imprescindible gestionar al personal de forma que permita la 
integración de todas: docencia, asesoría y gestión de la información en toda la universidad. La de docentes, al formar parte del curriculum académico asignaturas dedicadas a la Alfabetización Informacional el bibliotecario pasa, incluso, a compartir la docencia en el aula con los profesores. Es el caso de la Universidad Carlos III de Madrid en la que, en todos sus grados, los alumnos cursan una asignatura titulada "Técnicas de búsqueda y uso de la información" en el primer curso académico y donde los bibliotecarios, como especialistas en las distintas áreas de conocimiento, imparten sesiones de búsqueda y recuperación de la información (Aguilera et al., 2010). La de asesores, en temas como los de propiedad intelectual y derechos de autor. Y la función de gestor de la información en toda la universidad, no sólo en la biblioteca, al encargarse de la información de la web institucional y de las plataformas de aprendizaje utilizadas en la docencia. Todas estas nuevas funciones conllevan, por otra parte, una relación muy estrecha con los docentes e informáticos.

\subsection{CRISIS ECONÓMICA - REPERCUSIONES EN LA GESTIÓN PRESUPUESTARIA}

La crisis económica mundial que se inicia en el 2008 se manifiesta, como es obvio, en los recortes del gasto en las Administraciones públicas. Ello se traduce, en muchos casos, en una reducción del presupuesto para la cultura en general y, por ende, para las bibliotecas. Aunque en el año 2008 no se veía aún el alcance de la crisis, poco a poco van contando con menos recursos y se ven obligadas a disminuir algunas partidas pero a dar los mismos o incluso más servicios. Más aún si tenemos en cuenta que, ya antes de que la crisis fuera evidente, algunas bibliotecas como las públicas venían experimentando un incremento considerable en la demanda y prestación de servicios. Las cifras que ofreció el Departamento de la Generalitat de Cataluña en 2008 mostraban que las bibliotecas públicas y los bibliobuses catalanes habían incrementado el número de visitas en un $11,2 \%$ en relación con el año anterior. También en la Comunidad de Madrid el número de usuarios creció un 10,4\% en 2008 y el servicio de telebiblioteca, que facilita a personas con discapacidad y de la tercera edad el acceso a la lectura, un $30 \%$. Otras comunidades experimentaron igualmente incrementos similares (Colomer, 2009).

Algunas memorias anuales de bibliotecas universitarias reflejan el descenso del presupuesto. Por ejemplo, en la biblioteca de la universidad Carlos III, hasta el año 2008 , se produce una pequeña subida del $4 \%$ en las adquisiciones bibliográficas que permite, únicamente, hacer frente a la inflación. A partir del 2009 y hasta el momento actual se ha producido una reducción del 7,3\%, 15\% y $10 \%$, respectivamente cada año. Esto sucede incluso a pesar de la puesta en marcha de nuevas titulaciones. También otras instituciones del entorno universitario y científico se han visto obligadas a disminuir sus inversiones en los recursos 
informativos de cara al año 2011. En el Consejo Superior de Investigaciones Científicas (CSIC), Baquero (2010) advierte que en el 2011 las publicaciones periódicas serán las más afectadas por el recorte. Igualmente, el presupuesto de la Biblioteca Nacional de España sufrió una disminución en el 2010 de un 15,5\% en relación al 2009 (Salaberria, 2010). Lo mismo les ha sucedido a otro tipo de bibliotecas, de instituciones privadas y públicas, escolares, móviles.

La literatura profesional de este período relacionada con la crisis económica no es especialmente abundante teniendo en cuenta que sus efectos empiezan a notarse a partir del 2009. Coinciden, en términos generales, en la necesidad de demostrar, ahora más que nunca, la eficacia y la eficiencia de la biblioteca, así como justificar su función y valor social (Pérez Iglesias, 2009). Los trabajos se centran, además de mostrar la preocupación por la situación (González Fernández-Villavicencio y Calderón Rehecho, 2010), en dar recomendaciones e iniciativas a seguir para sortear la crisis (Reina Segovia, 2009 y Gómez Hernández, 2010 a y b). Entre otras, la necesidad de poner en marcha una planificación estratégica de calidad (Reina Segovia, 2010), demostrar la eficiencia del gasto en los recursos de información que se adquieren (Rodríguez Bravo y Olea, 2010), buscar fuentes de financiación alternativas (Pérez Pulido, 2010), fomentar la creatividad y la innovación (Riaza Chaparro, 2010) y, por supuesto, cooperar (González Fernández-Villavicencio y Calderón Rehecho, 2010).

Conocer la repercusión que la crisis ha tenido en las bibliotecas ha sido objetivo de algunos grupos de profesionales interesados en este debate:

- El Foro de Especialistas en Información y Documentación de Andalucía, reunidos en mayo del 2011, extrajo como conclusión destacada la de considerar la crisis como una oportunidad para las bibliotecas si se reacciona y readaptan ágilmente los servicios priorizando los dirigidos a los colectivos más afectados (AAPID, 2011).

- El grupo de trabajo integrado por miembros de la Asociación Andaluza de Bibliotecarios presentó un estudio del impacto de la crisis económica en las bibliotecas andaluzas en el último Congreso de FESABID, celebrado en mayo del 2011, partiendo de los datos obtenidos a partir de un cuestionario, y cuyas conclusiones (Gutiérrez Santana, Real Duro, Bustamante y Guerrero Salguero, 2010) revelan que la crisis está afectando a las bibliotecas, especialmente en la reducción del presupuesto para adquisiciones y en la paralización de proyectos, pero sólo el $18 \%$ de los encuestados tiene previsto llevar a cabo un plan de actuación para hacerle frente. Las medidas tienen que ver con la reducción de gastos, el fomento de las actividades sin presupuesto, la colaboración con otras instituciones, la planificación y el cobro de algunos conceptos, entre otras. 


\section{TEMAS EMERGENTES QUE INFLUYEN EN LA GESTIÓN DE LAS BIBLIOTECAS}

A los temas ya referidos que han sido objeto de mayor preocupación se añaden otros que han irrumpido con fuerza a lo largo de este período y que, de igual modo, atañen y se relacionan con la gestión de las bibliotecas:

\subsection{RESPONSABILIDAD SOCIAL CORPORATIVA}

La demanda de la sociedad de comportamientos socialmente responsables ha llevado a las empresas a aplicar criterios de Responsabilidad Social Corporativa. Al igual que las empresas, las bibliotecas también han visto la necesidad de emplear estos criterios e introducir estas prácticas. Lo han hecho secundando, en ocasiones, las directrices de la institución de la que dependen. Es el caso de algunas universidades en las que se han creado unidades y comisiones de Medioambiente y Sostenibilidad para marcar criterios de actuación para todos los servicios. Merece destacarse el esfuerzo de bibliotecas, como la universitaria de Burgos, por haberse empleado en estas prácticas sin la guía y el marco de referencia de su institución (Sedano Ruiz, 2010).

Los dos compromisos a los que se han unido las bibliotecas han sido objetivo de las III Jornadas profesionales de la Red de bibliotecas del Instituto Cervantes, celebradas en diciembre de 2010:

- La sostenibilidad medioambiental, convertida, por otra parte, en un nuevo grupo de interés especial en la IFLA (Miner Van Neygen, 2010). Esta línea de actuación se traduce en la puesta en marcha de objetivos ligados, por ejemplo, al ahorro de papel y de espacio, mediante el paso de la colección impresa a formato electrónico, al ahorro de energía fomentando el uso del correo electrónico y la intranet, además de la gestión de los residuos como el tóner, pilas, etc. (Mesa Sánchez, 2010).

- La cooperación al desarrollo de las bibliotecas se manifiesta en las acciones de formación, investigación, asistencia técnica a proyectos en el exterior, difusión y sensibilización. En el año 2006 la Comisión de Cooperación al desarrollo de la CRUE, integrada por 24 universidades, aprueba el Código de conducta en materia de cooperación al desarrollo, permitiendo y facilitando la actuación del personal de Administración y Servicios de las universidades como agentes de desarrollo en el terreno. En este contexto, las bibliotecas universitarias españolas vienen colaborando en países de Hispanoamérica, África y Asia, fundamentalmente, en la creación o mejora de bibliotecas, actividades de animación a la lectura, en temas relacionados con la investigación, conservación y preservación de fondos y en la creación de sistemas bibliotecarios (García Martín, 2010). 
Las últimas Jornadas de SEDIC, celebradas en marzo del 2011, se dedicaron a la Responsabilidad Social Corporativa. En ellas se expusieron las diferentes formas de entenderla con prácticas concretas puestas en marcha en empresas y en la universidad. Esta última estuvo representada por la Biblioteca Nacional y las bibliotecas de la Universidad Politécnica y la Complutense de Madrid (SEDIC, 2011).

Al tema de la sostenibilidad medioambiental se suma otro que empieza a cobrar una nueva dimensión ante los riesgos que amenazan al bibliotecario en su actividad diaria, la ética profesional. Nos referimos, por ejemplo, a los casos de censura e intromisión en las tareas propias de la profesión, acontecidos no hace mucho en las bibliotecas públicas de Navarra.

Algunos docentes han justificado la enseñanza de esta materia y han hecho hincapié en la formación en los valores y el compromiso cívico que deben recibir los futuros profesionales de las bibliotecas (Pérez Pulido 2004; López López, 2009). El Libro Blanco de la titulación en Información y Documentación propone la Ética y la Deontología profesional como contenido común de los planes de estudio. Y, acorde con ello, algunos planes de estudio en los nuevos grados adaptados al Espacio Europeo de Educación Superior cuentan ya con una asignatura de formación básica dedicada a esta materia. No obstante, a nivel profesional, hay una cuestión pendiente y es la falta de un código ético que represente los valores de los profesionales de la información españoles. En el seno de la SEDIC, en noviembre del 2010, Paz Fernández, directora de la biblioteca de la Fundación Juan March, impulsó la creación de un Grupo de Trabajo para investigar y abordar la elaboración de un código que sirviera de base para todos los colectivos relacionados con la profesión. Los primeros resultados del grupo, después de realizar una encuesta entre los profesionales, se han expuesto recientemente en las últimas jornadas FESABID (Grupo de Trabajo de Ética, 2011). Con el código se pretende que los profesionales cuenten con una herramienta que les ayude a tomar decisiones, abordar los conflictos que puedan surgir y formular soluciones adecuadas a cada caso, además de justificarlas.

\subsection{LOS REPOSITORIOS INSTITUCIONALES}

Desde que se pone en marcha el movimiento Open Access, con la Declaración de Budapest en el 2002, los repositorios institucionales han ido creciendo paulatinamente y con ello la necesidad de gestionarlos adecuadamente al contener las colecciones digitales propias de la institución. Los trabajos intentan, desde el inicio, ayudar a comprender en qué consiste la Iniciativa de los Archivos Abiertos (OIA), cómo es y cómo puede ayudar a la divulgación de la información científica animando a las instituciones científicas a poner en marcha proyectos de este tipo (Bueno de la Fuente, 2007). Las investigaciones centradas en los repositorios se ciñen, fundamentalmente, al ámbito de las bibliotecas universitarias y científicas, 
como responsables directas de su gestión al pasar a formar parte de la colección los recursos que contienen. No obstante, hay excepciones que analizan el caso de las bibliotecas nacionales (Rodríguez Bravo, 2007). También en esta ocasión el tema de la propiedad intelectual está presente y ha sido una preocupación constante desde el inicio (Vives, 2005). En la línea de conseguir una gestión adecuada, se recomiendan los pasos y tareas que debe seguir la biblioteca al liderar estas iniciativas (Hernández, Rodríguez y Bueno, 2007). Los factores clave para el éxito de los repositorios se han analizado apuntando que se requiere, por parte de la biblioteca, una buena labor informativa entre los autores sobre las ventajas, los procedimientos a seguir para depositar los trabajos e, incluso, ofertar servicios que ayuden a hacerlo y promocionar el autoarchivo (Keefer, 2007; Melero, 2007). A estos trabajos hay que añadir los que exponen el panorama de los repositorios institucionales en nuestro país (Melero, 2008 y Melero et al, 2009). Los más recientes, partiendo de la situación actual, tratan de elaborar una serie de directrices que guíen su creación y permitan la evaluación (Barrueco Cruz, 2010).

\section{TEMAS RECURRENTES}

En materia de gestión, hay una serie de temas que vienen siendo objeto de atención reiterada con anterioridad al período fijado en este trabajo y que continúan siéndolo hasta el momento actual. Siguen respondiendo a la necesidad de continuar mejorando y demostrar el rendimiento de las bibliotecas, por una parte, $y$, por otra, al desempeño de las nuevas tareas, funciones y servicios que han tenido que implementar:

\subsection{CALIDAD, EVALUACIÓN Y PLANIFICACIÓN.}

Desde el bienio 1992-94 en el que se introduce la evaluación de la calidad en la Universidad Española, estos temas se convierten en los más tratados dentro del panorama de la gestión bibliotecaria. En el 2005 se puede empezar a hablar de consolidación de la calidad en las bibliotecas, dado que, algunas bibliotecas universitarias, inician ya su segundo proceso de evaluación después de haber obtenido por el primero un reconocimiento a la calidad a través de distintas vías (Balagué, 2007). Algunos trabajos de este período, a modo de recopilación de lo realizado, ofrecen estados de la cuestión (Pinto, Balagué y Anglada, 2007; Jorge García-Reyes, 2008). A partir del 2006 las bibliotecas consiguen los primeros reconocimientos europeos a la calidad. Se puede considerar la internacionalización del proceso. Empezamos a contar con bibliotecas con sellos de excelencia, como reconocimiento a su buena gestión, tema que marcará la línea de algunos trabajos (Rico y Calvo, 2010). Las herramientas propias de la calidad, como las cartas de servicios (Alós et al, 2010) o determinados modelos de encuestas (Ferrer-Torrens y Rey, 2005 y 
Pinto, Fernández Marcial y Gómez Camarero, 2007) son otra constante a lo largo de esta etapa y han sido objeto de tratamiento monográfico. Asimismo, la adopción de técnicas de gestión íntimamente ligadas a la calidad, como el benchmarking, se muestran a través de aplicaciones prácticas (Lopez-Gijón et al. , 2010).

La evaluación viene siendo tema de atención preferente entre los profesionales y docentes dedicados a la gestión, de ahí que aspectos como la medición del impacto y rendimiento continúan estando presentes conforme avanza el tiempo (Bustos, 2007, Amat de la Flor et al., 2006). Nuevas propuestas de aplicación y de indicadores revelan la necesidad de adaptación constante de las herramientas ante los cambios (Borrego y Urbano, 2005; Mano y Albelda, 2009; García García, Berbes Cardos y Gimenó Perelló, 2010).

Las primeras experiencias en evaluación durante el bienio 1996-98 terminan en la elaboración de los correspondientes planes de mejora que, a su vez, darán lugar a los primeros planes estratégicos. El proceso seguido y la implementación de estos planes han sido objeto de seguimiento por algunos profesionales (GarcíaReche, 2008). Los dos planes estratégicos de REBIUN, con años enmarcados en este período, son un ejemplo del avance de la planificación estratégica en las bibliotecas universitarias.

También las acciones de planificación de bibliotecas, a nivel ministerial, se han analizado para demostrar que no existe una política bibliotecaria estatal (Menéndez Seoane, 2007). La situación no es la misma en otras demarcaciones territoriales, como ocurre en la provincia de Barcelona, aunque son deseables acciones de mejora en relación con lo realizado (Omeya, Permanyer y Vilagrosa, 2009).

En este epígrafe es de rigor añadir la monografía editada por Luisa Orera (2005) que, centrada en el ámbito de las bibliotecas universitarias, ofrece capítulos dedicados a estas técnicas: planificación, evaluación, etc. También la obra de Juan J. Fuentes Romero (2007) trata los aspectos teóricos y prácticos de la planificación.

\subsection{GESTIÓN DEL CONOCIMIENTO}

Este nuevo modelo de gestión que emerge en los años 90 del siglo pasado, asociado en exceso a las herramientas tecnológicas en sus inicios, ha pasado a centrarse más en los recursos humanos, demostrando así que la tecnología es tan sólo un medio y las personas son su factor clave de éxito. Algunas experiencias basadas en metodologías como las "comunidades de práctica" aplicadas en bibliotecas demuestran la importancia del factor humano en la consolidación de esta técnica de gestión (Sánchez Galán, 2009).

Aunque los trabajos en su mayoría se centran en experiencias relativas a la implementación de proyectos, conviene citar en el período al que se refiere este trabajo la aparición del libro de Pérez-Montoro Gutiérrez (2008) por recoger los 
fundamentos teóricos de la técnica, así como ejemplos de aplicaciones prácticas en las que viene a demostrar la dificultad que supone gestionar el conocimiento individual para ponerlo a disposición de la colectividad. Además de tratar otros temas, la monografía de Roser Lozano (2007), teniendo como marco de actuación la biblioteca pública y la participación activa de los ciudadanos, propone también, entre otras ideas clave, la gestión del conocimiento.

\subsection{GESTIÓN DE LA COLECCIÓN}

Ya se ha hecho referencia a la repercusión del libro electrónico en la gestión de la colección como tema "estrella", así como a la gestión de los repositorios institucionales. Además, hay que añadir otros que venían ya siendo objeto de tratamiento anterior y que tienen que ver con las funciones asociadas a la gestión de la colección:

\subsubsection{GESTIÓN DE LA COOPERACIÓN}

Teniendo en cuenta que, desde 1997, la cooperación ha sido objeto de análisis a través de las jornadas que se vienen celebrando bajo ese título en nuestro país, es posible valorar el interés que tiene el tema, así como la necesidad de que cada biblioteca gestione las iniciativas de cooperación en las que participa y valore adecuadamente las nuevas acciones que estaría dispuesta a emprender. La consulta de las actas de estas jornadas da una buena idea de la situación española en esta materia. La cooperación, no sólo entre bibliotecas sino también entre estas y otras instituciones, se ve como una solución eficaz para dar muchos servicios bibliotecarios. Esa razón hace, posiblemente, que en muchos trabajos se expongan los beneficios para las bibliotecas (Termens, 2005) e, incluso, se demuestre su eficacia a través de ejemplos concretos de actuación (Martín-Montalvo Cortés, Valverde Facal e Iglesia Sánchez, 2010; Muñoz Muñoz y Argente Jiménez, 2010).

Una visión actualizada del tema, desde la perspectiva de actuación del Ministerio de Cultura, se puede ver en el trabajo de Carrato Mena (2010) en el que la cooperación se presenta para las bibliotecas públicas como un reto para la creación de recursos digitales.

Para el caso de las bibliotecas universitarias, la tesis doctoral de Blanca San José (2011), la cooperación se muestra como un factor clave de éxito para la gestión de la colección en este momento.

\subsubsection{GESTIÓN DE LA PRESERVACIÓN DIGITAL}

La eclosión de proyectos implementados en las bibliotecas revela el auge e interés que suscita este tema en función del número de trabajos en los que se dan a conocer. La tesis doctoral de Bárbara Múñoz de Solano y Palacios, dedicada a la 
preservación digital y defendida en el 2005 ofrece, en este período, un completo panorama de la situación. Algunos aspectos de su investigación se exponen en distintos artículos actualizados en los que aborda, entre otras cuestiones, los costes (Múñoz de Solano y Palacios, 2006 a) y las peculiaridades de tipo organizativo que conlleva (Múñoz de Solano y Palacios, 2006 b). En el 2007, A. Keefer y Nuria Gallart, realizan de nuevo una puesta al día del tema, además, desde la perspectiva de la gestión. Con posterioridad se han realizado otros estados de la cuestión apropiados al momento (Carrato Mena, 2008 y Termens, 2009) que revelan cómo esta función continua siendo un reto para las bibliotecas.

\subsubsection{GESTIÓN DE LA PROPIEDAD INTELECTUAL Y DE LOS DERECHOS DE AUTOR}

Íntimamente ligado al tema anterior, de ahí que se traten juntos en numerosos trabajos, la gestión de la propiedad intelectual y de los derechos de autor continúa siendo un conflicto para las bibliotecas, fundamentalmente, desde que las bibliotecas digitales son una realidad. La legislación no parece estar en consonancia con la preservación, obviando los problemas que la afectan (Fernández Molina, 2010). El papel que tienen las bibliotecas en la gestión de la propiedad intelectual y de los derechos de autor pasa, en este momento, por ofrecer servicios de asesoría (Lorenzo Escolar, 2009), tal y como pueden comprobar los usuarios de algunas bibliotecas universitarias que cuentan con ellos.

\section{CONCLUSIONES}

La identificación de tendencias y su correspondiente análisis permite hacer una serie de afirmaciones:

- Resulta harto complicado prescindir de algunos temas que podían formar parte de este análisis de tendencias, teniendo en cuenta que deben ser pocos los elementos que no inciden o repercuten en la gestión de una biblioteca. Cualquier servicio nuevo, actividad o función tiene una clara influencia en la gestión, puesto que obliga a destinar algún tipo de recurso para gestionarlo. Por otra parte, las instituciones de las que dependen las bibliotecas se encuentran en un mundo competitivo y en constante cambio enfrentándose a los mismos retos que el resto de las organizaciones, lo que se traduce también en cambios que las bibliotecas deben gestionar.

- El conjunto de aspectos que se han tratado muestran que, en este momento, los que se dedican a la gestión en las bibliotecas tienen que rodearse de un equipo multidisciplinar de expertos en temas muy diversos (redes sociales, dispositivos móviles, contenidos en abierto, habilidades de negociación y contratación, comunicativas, dominio de marcos legales, informática, por citar algunos) además de tener conocimiento de las técnicas de gestión necesarias 
para maximizar los recursos con los que cuentan y obtener el mejor rendimiento posible).

- Preocupan y están presentes en los trabajos de este período los mismos temas de interés que afectan a las bibliotecas de otros países, si tenemos en cuenta, por ejemplo, el artículo de la ACRL (2010) relativo a las tendencias en las bibliotecas universitarias para el 2010.

- Es significativo el número de trabajos que coinciden en el mismo tema y año, así como en la forma de abordarlo. Los relacionados con el libro electrónico, los CRAIs y la calidad de los servicios reflejan esta casuística.

- Las bibliotecas universitarias son las más vinculadas a los estudios relacionados con la aplicación de técnicas de gestión aunque la crisis ha llevado a implementarlas por primera vez en otras bibliotecas como algunas de las públicas.

- La crisis económica ha obligado a realizar cambios en la gestión presupuestaria ante la reducción de los presupuestos. Los bibliotecarios responden a esta situación tratando de buscar mejores sistemas de reparto, buscando más fuentes de financiación, aplicando técnicas como la planificación $\mathrm{y}$, en algunos casos, evaluando la colección con mayor frecuencia y de manera más exhaustiva para liberar recursos que les permitan hacer frente a necesidades más prioritarias. Un aspecto positivo de la crisis es que ha hecho recordar e incidir en el papel social de las bibliotecas y el valor que tienen para la sociedad, posiblemente debido a que, a pesar de la mala situación económica en la que vivimos, las bibliotecas han tenido que hacer frente a una mayor demanda social.

- La técnica de gestión que se ha visto más afectada en este período es el marketing, al verse renovada, compartida y cobrado una nueva dimensión al hacer uso de las nuevas herramientas de la web 2.0 y las redes sociales.

- Ante la crisis, la cooperación se antoja una necesidad para las bibliotecas y no sólo entre las bibliotecas sino con otras instituciones con las que puedan establecerse sinergias y ayudas mutuas. Y, por supuesto, se apuesta y se trata de fomentar la innovación y la creatividad en los centros de trabajo tal y como han recomendado algunas profesionales como Roser Lozano y Nieves González. Más aún, contando con las facilidades que proporcionan las herramientas y principios de la web 2.0 y las redes sociales.

\section{REFERENCIAS BIBLIOGRÁFICAS}

ACRL. "2010 top ten trends in academic libraries: A review of the current literature". College \& Research Libraries News, June 2010, 71, p. 286-292.

AGUILERA, R. et al. "Enseñando a buscar y a utilizar la información estadística: una colaboración conjunta entre docentes y personal de la biblioteca" [en línea]. En World 
Library and Information Congress: 76th IFLA General Conference and Assembly. Gothenburg, $\quad$ Sweden, $2010 . \quad$ Disponible en: $\mathrm{http}$ //www.ifla.org/files/hq/papers/ifla76/86-aguilera-es.pdf [consulta: 7 junio 2010].

ALONSO ARÉVALO, J; CORDÓN GARCÍA, J.L. y GÓMEZ DÍAZ, R. (a) "El libro electrónico en la biblioteca universitaria y de investigación" [en línea]. Biblios, 2011, 42. Disponible en: http://eprints.rclis.org/bitstream/10760/15537/1/ Biblioos\%20-\%20Alonso.pdf [consulta: 3 junio 2011].

ALONSO ARÉVALO, J; CORDÓN GARCÍA, J.L. y GÓMEZ DÍAZ, R. (b) "Libros electrónicos: oferta comercial y redes P2P" [en línea]. El profesional de la información, 2011, v. 20, n. 2, p. 141-150. Disponible en: http://anatomiteca.com/wp-content/uploads/2011/03/Cordon-GomezAlonso.pdf [consulta: 10 junio 2011].

ALÓS, J.; CABO, M.; ESPINÓS, M.; GARRETA, M. y GRANÉ, T. "Un compromiso de servicio común: la carta de servicios de la biblioteca y del servicio de informática de la Universitat Pompeu Fabra" [en línea]. En II Jornadas de calidad y bibliotecas. Málaga, REBIUN, 2010. Disponible en: http://www.biblioteca.uma.es/ bbldoc/2JornadasCalidad2010/actas/7CartaServiciosUPF.pdf [consulta: 10 junio 2011].

ALVAREZ MARAÑÓN, G. "Amenazas 2.0 para la Biblioteca 2.0" [en línea]. En $V$ Congreso Nacional de Bibliotecas Públicas. Gijón 2010. Disponible en: http://www.mcu.es/bibliotecas/docs/MC/2010/CongresoBP/GonzaloAlvarez.pd f [consulta: 3 junio 2011].

ALVITE DÍEZ, M.L. y RODRÍGUEZ BRAVO, B. "Colecciones de libros electrónicos en las bibliotecas universitarias españolas" [en línea]. En VIII Jornadas de Gestión de la Información, Madrid, 2006. Disponible en: http://eprints.rclis.org/bitstream/10760/8484/1/Sesion_3_comunicacion_1_Alvi te.pdf [consulta: 10 junio 2011].

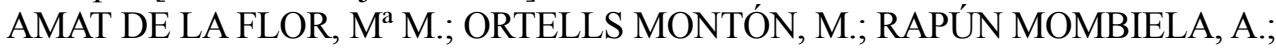
LATORRE ZACARÉS, I. «Estudio de rentabilidad de la biblioteca pública provincial de Valencia». El profesional de la información, 2006, v. 15, n. 5, pp. 342-351.

ARQUERO AVILÉS, R. et al. "Planificación de Centros de Recursos para el Aprendizaje y la Investigación (CRAI's): un estudio de caso. Grupo "CRAI-Universidad Complutense de Madrid". Boletín de la ANABAD, 2007, 57,2, p. 335-357.

ASOCIACIÓN ANDALUZA DE PROFESIONALES DE LA INFORMACIÓN Y LA DOCUMENTACIÓN (AAPID). Las bibliotecas en tiempo de crisis 2011 [en línea]. Disponible en: http://www.aapid.org/website/ index.php?option

$=$ com_content $\&$ view $=$ article $\& \mathrm{id}=53:$ bibliocrisis $\&$ catid $=21:$ noticias $\&$ Itemid $=27$ [consulta: 3 junio 2011].

BALAGUÉ, N. "La biblioteca universitaria: centro de recursos para el aprendizaje 
y la investigación. Una aproximación al estado de la cuestión en España" [en línea]. En I Jornadas Rebiun, 2003. Disponible en: http://biblioteca.uam.es /sc/documentos/Jornadas_REBIUN/3\%20\%20biblioteca_universitaria_CRAI.pdf [consulta: 3 junio 2011].

BALAGUÉ, N. "Consolidando la calidad en las bibliotecas universitarias: evaluaciones, sellos, diplomas y certificaciones". El profesional de la información, 2007, julio-agosto, v. 16, n. 4, p. 338-342.

BAQUERO, M. "La evaluación de la colección de la biblioteca, un bien necesario en tiempos de crisis" [en línea]. Enredadera. Boletín electrónico de la red de bibliotecas del CSIC, julio 2010, n. 18. Disponible en: http://bibliotecas.csic.es/enredadera/boletin18/crisis.html [consulta: 3 junio 2011].

BARRUECO CRUZ, J.M. (coord.) Guía para la evaluación de los repositorios institucionales de investigación. Madrid: CSIC, 2010. Disponible en: http://digital.csic.es/bitstream/10261/35735/1/GuiaEvaluacionRecolectav1.01.pdf [consulta: 10 junio 2011].

BORREGO, Á. y URBANO, C. "Estadísticas e indicadores de rendimiento de colecciones y servicios bibliotecarios de carácter electrónico: estudio de caso de las revistas electrónicas". El profesional de la información, 2005, v. 14, n. 2, p. 30-38.

BUENO DE LA FUENTE, G. La iniciativa de archivos abiertos (OAI): situación y perspectivas en España y Latinoamérica. Bogotá: Rojas Eberhard, 2007.

BUSTOS GONZÁLEZ, A. "Bibliotecas universitarias: ¿sabemos medir sus resultados e impactos?" El profesional de la información, 2007, v. 16, n 4, p. 281-286.

CABRÉ SERRA, A. "Biblioteques de Barcelona en las redes sociales" [en línea]. En $V$ Congreso Nacional de Bibliotecas Públicas. Gijón 2010. Disponible en: http://www. $\quad$ mcu.es//bibliotecas/docs/MC/2010/CongresoBP/AnnaCabre.pdf [consulta: 3 junio 2011].

CARRATO MENA, M.A. "Las colecciones digitales en España: situación actual y perspectivas de futuro". El profesional de la información, 2008, v. 17, n. 4, p. 418421.

CARRATO MENA, M.A. "Cooperación bibliotecaria para un acceso global al conocimiento". El profesional de la información, 2010, v. 19, n. 5, p. 449-454.

CASAL REYES, M.I. "El CRAI y nuevos retos de las bibliotecas universitarias". En I Jornadas Narón. Nuevas tecnologías en bibliotecas y archivos (Noviembre 2010). A Coruña: Universidad, 2011, p. 89-117.

CLAVERO, J.; CODINA, M.; PÉREZ, A. Y SERRAT-BRUSTENGA, M. "Estudio de caso de servicio de préstamo de libros electrónicos". El profesional de la información, 2009, v. 18, n. 2, p. 237-242.

COLOMER, A. "Las bibliotecas resurgen con la crisis" [en línea]. El mundo.es, 7 Junio 2009. Disponible en: http://www.elmundo.es/suplementos/ magazine/2009/506/1244105626.html [consulta: 3 junio 2011]. 
CORDÓN GARCÍA, J.A. y ALONSO ARÉVALO, J. "Las políticas de adquisición de libros electrónicos en bibliotecas: licencias, usos y derechos de autor" [en línea]. En $V$ Congreso Nacional de Bibliotecas Públicas, Gijón 2010. Disponible

en: http://www.mcu.es/bibliotecas/docs/MC/2010/CongresoBP/1AntonioCordonJul ioAlonso.pdf [consulta: 3 junio 2011].

CORDÓN GARCÍA, J.A.; ALONSO ARÉVALO, J. y MARTÍN RODERO, H. "Los libros electrónicos: la tercera ola de la revolución digital". Anales de Documentación, 2010, 13, p.53-80.

CORDÓN GARCÍA, J.A.; GÓMEZ DÍAZ, R. Y ALONSO ARÉVALO, J. Gutemberg 2.0. La revolución de los libros electrónicos. Gijón: Trea, 2011.

COSTA KNUFINKE, J. "Adaptación de las editoriales españolas al libro electrónico". El profesional de la información, 2010, v.19, n. 1, p. 13-20.

DOMÍNGUEZ AROCA, M.I. "La biblioteca universitaria ante el nuevo modelo de aprendizaje: docentes y bibliotecarios, aprendamos juntos porque trabajamos juntos" [en línea]. RED. Revista de educación a distancia, 2008. Disponible en: http://www.um.es/ead/red/M4/dominguez9.pdf [consulta: 3 junio 2011].

FERNÁNDEZ MOLINA, J.C. "Preservación digital y derechos de autor ¿un conflicto sin solución?" [en línea]. En $V$ Congreso Nacional de bibliotecas públicas. Gijón, 2010.

http://travesia.mcu.es/portalnb/jspui/bitstream/10421/4902/2/PPTJuanCarlosFerna ndez.pdf [consulta: 10 junio 2011].

FERRER TORRENS, A. y REY MARTÍN, C. "Aplicación del LIBQUAL+ en el CRAI de la Universidad de Barcelona" [en línea]. En IX Jornadas Españolas de Documentación. Madrid, 2005, v. 68, 3. Disponible en: http://diposit.ub.edu/dspace/bitstream/2445/11479/1/libqual.pdf [consulta: 9 junio 2011].

FUENTES ROMERO, J.J. Planificación de centros documentarios. Organización y funcionamiento de bibliotecas, centros de documentación y centros de información. Gijón: Trea, 2007.

GARCÍA GARCÍA, J.; BERBES CARDOS, J.A.; GIMENO PERELLÓ, J. "Biblioteca Complutense de Madrid: evaluando cargas de trabajo para la mejora del servicio público" [en línea]. En II Jornadas Universitarias de Calidad y Bibliotecas, Málaga del 20 al 21 de mayo de 2010. Disponible en: http://dspace.uma.es/xmlui/

bitstream/handle/10630/4178/2BCM.pdf? sequence=1 [consulta: 9 junio 2011].

GARCÍA JIMÉNEZ, D. "Redes sociales: posibilidades de Facebook para las bibliotecas públicas" [en línea]. Biblioteconomia i Documentació, 2010, 24, Disponible en: http://www.ub.edu/bid/24/garcia2.htm [consulta: 3 junio 2011].

GARCÍA MARCO, F.J. "El libro electrónico y digital en la ecología informacional". El profesional de la información, 2008, Vol. 17, Nº 4, p. 373-389.

GARCÍA MARTÍN, A. "Las bibliotecas y la cooperación al desarrollo" [en línea]. 
En III Jornada profesional de la Red de bibliotecas del Instituto Cervantes. Madrid, 16 de diciembre de 2010. Disponible en: http://www.cervantes.es/ imagenes/File/biblioteca/jornadas/jornada_3/documentacion/garcia_martin_ara celi.pdf [consulta: 3 junio 2011].

GARCÍA RECHE, G. "Elementos del sistema de gestión de la calidad en una biblioteca universitaria" [en línea]. En XI Jornades Catalanes d'Informació $i$ Documentació. Barcelona, 2008. Disponible en: http://dspace.uma.es/xmlui/ bitstream/handle/10630/2740/GarciaRecheElementosCalidad.pdf?sequence=2 [consulta: 9 junio 2011].

GÓMEZ HERNÁNDEZ, J.A. "Las bibliotecas públicas ante la recesión económica". Educación y biblioteca, 2010, n. 178, p. 68-74.

GÓMEZ HERNÁNDEZ, J.A. "La acción de las bibliotecas públicas en tiempos de crisis" [en línea]. En Notas ThinkEPI 2010. Disponible en: $\mathrm{http} / / /$ www.thinkepi.net/accion-bibliotecas-publicas-tiempos-crisis

[consulta: 3 junio 2011].

GONZÁLEZ FERNÁNDEZ-VILLAVICENCIO, N. "Bibliotecas y marketing en red" [en línea]. BiD: textos universitaris de biblioteconomia i documentació, 2009, n. 23. Disponible en: http://www.ub.edu/bid/23/gonzalez2.htm [consulta: 10 junio 2011]

GONZÁLEZ FERNÁNDEZ-VILLAVICENCIO, N. "Responsabilidad social enredada de las bibliotecas universitarias". Mi biblioteca: la revista del mundo bibliotecario, 2010, 20, p. 60-65.

GONZÁLEZ FERNÁNDEZ-VILLAVICENCIO, N. "Communitiy manager: ¿dónde termina el marketing y comienza la biblioteca?" [en línea]. En 19 Jornadas infantiles, juveniles y escolares. Fundación Germán Sánchez Ruipérez, Salamanca, junio 2011. Disponible en: http://www.fundaciongsr.es/wfuns/activos/texto/ wfuns_informacion_0620RprpX9rKbDW90MRQ.pdf [consulta: 15 junio 2011].

GONZÁLEZ FERNÁNDEZ-VILLAVICENCIO, N. y CALDERON REHECHO, A. "Peligra tu puesto de trabajo?" Bibliotecas universitarias en tiempos de crisis. Educación y Biblioteca, 2010, n. 178, p. 101-106.

GRUPO DE TRABAJO DE ÉTICA DE SEDIC. "Hacia un código deontológico común: resultados de la encuesta" [en línea]. En XII Jornadas Españolas de Documentación, Mayo 2011. Disponible en: http://www.sedic.es/Fesabid-2011-codigo-deontologico.pdf [consulta: 3 junio 2011].

GUTIÉRREZ SANTANA, F.; REAL DURO, A; BUSTAMANTE RODRÍGUEZ, A.T. y GUERRERO SALGUERO, C. "Estudio sobre el impacto de la crisis económica en las bibliotecas andaluzas" [en línea]. Boletín de la Asociación Andaluza de Bibliotecarios, 2010, n. 100, p. 119-136. Disponible en: http://www.aab.es/aab/images/stories/Boletin/100/7_impacto_crisis_economica .pdf [consulta: 3 octubre 2011].

HERNÁNDEZ PÉREZ, T.; RODRÍGUEZ MATEOS, D. BUENO DE LA 
FUENTE, G. "Open Access: el papel de las bibliotecas en los repositorios institucionales de acceso abierto". Anales de Documentación, 2007, vol. 10, p. 185-204.

JORGE GARCÍA-REYES, C. "Acreditación y certificación en bibliotecas universitarias: la experiencia española" [en línea]. En Coloquio Administración y Liderazgo en el Campo Informativo. Satisfacción de usuarios: Evaluación integral de bibliotecas. Universidad Veracruzana, Veracruz, 2008. Disponible en: http://www.uv.mx/usbi_ver /alci08/docs/c6 carmen jorge.pdf [consulta: 9 junio 2010].

JUNQUERA TEMPRANO, J. Políticas y líneas de apoyo de la Administración General del Estado [en línea]. En El libro electrónico. Grupo de Trabajo sobre El Libro Electrónico. Observatorio de la Lectura y el Libro. Abril 2010. Disponible en: http://www.mcu.es/libro/docs/MC/Observatorio/pdf/LIBRO_ELECTRONICO 2010.pdf [consulta: 3 junio 2011].

KEEFER, A. "Los repositorios digitales universitarios y los autores". Anales de Documentación, 2007, n. 10, p. 205-214.

KEEFER, A. Y GALLART, N. La preservación digital de recursos digitales. El reto para las bibliotecas del siglo XXI. Barcelona: UOC, 2007.

LARRAÑAGA RUBIO, J. "El mercado del e-book en España" [en línea]. Documentación de las ciencias de la información, 2008, vol. 31, p. 183-209. Disponible en: http://www.ucm.es/BUCM/revistas/inf/02104210/articulos/ DCIN0808110183A.PDF [consulta: 3 junio 2011].

LÓPEZ GIJÓN, J.; ÁVILA-FERNÁNDEZ, B.; PÉREZ-GÁLVEZ, I.J.; HERRERA-VIEDMA, E. "La calidad en las bibliotecas universitarias biomédicas según sus usuarios". El profesional de la información, 2010, v. 19, n. 3, pp. 255-259.

LÓPEZ LÓPEZ, P. "Compromiso cívico y formación inicial en biblioteconomía y documentación" [en línea]. En: García Caro, C. y Vílchez Pardo, J. (coord.). Homenaje a Isabel de Torres Ramírez: estudios de Documentación dedicados a su memoria. Granada: Universidad de Granada, 2009, p. 381-401. Disponible en: http://eprints.rclis.org/bitstream/10760/14125/1/HOMENAJE_I_TORRESPEDRO_LOPEZ_LOPEZ.pdf [consulta: 10 junio 2011].

LORENZO ESCOLAR, N. "La ley de propiedad intelectual y su repercusión en la actividad de las bibliotecas". Revista española de Documentación Científica, 2009 , v. 32, n. 4, p. 34-45.

LOZANO-DÍAZ, R. La biblioteca pública del S. XXI: atendiendo clientes, movilizando personas. Gijón: Ediciones Trea , 2006.

MANO GONZÁLEZ, M. DE LA Y ALBELDA-ESTEBAN, B. "Evaluación de bibliotecas nacionales: elaboración de una normativa internacional y su aplicación en la Biblioteca Nacional de España". El profesional de la información, 2009, v. 18, n. 6, p. 660-668. 
MARGAIX ARNAL, D. "Conceptos de web 2.0 y biblioteca 2.0: origen, definiciones y retos para las bibliotecas actuales". El profesional de la información, 2007, v. 16, n. 2, p. 95-106.

MARGAIX-ARNAL, D. "Lectura, Universidad y Recursos 2.0". Educación y Biblioteca, 2008, p. 83-88.

MARTÍN-GONZÁLEZ, J.C. y PIVETTA, E. "Factores clave en el proceso de adquisición de libros electrónicos". El profesional de la información, 2008, v.17, n. 4, p. 408-413.

MARTÍN-MONTALVO-CORTÉS， I.; VALVERDE-FACAL， M.V.; DE-LAIGLESIA-SÁNCHEZ, Y. "Cooperación en la Red de Bibliotecas del Instituto Cervantes. Dos ejemplos de actuación". El profesional de la información, 2010, v. 19 , n. 5, pp. 486-491.

MARZAL GARCÍA-QUISMONDO, M.A. "CRAI para la Educación en la Sociedad del Conocimiento: modelos ALFIN para la función educadora de los bibliotecarios" [en línea]. En IV Jornadas CRAI. Experiencias en el ámbito de la organización y convergencia de servicios. Burgos, 2006. Disponible en: http://www.rebiun.org/eventos/crai/ivcrai.html [consulta: 10 junio 2011].

MELERO, R. "Políticas sobre el libre acceso a la producción científica y la respuesta de los autores" [en línea]. En IV Congreso de Comunicación Social de la Ciencia, Madrid, 2007. Disponible en: http://eprints.rclis.org/bitstream/ 10760/10429/1/Melero-CSciencia.pdf [consulta: 10 junio 2011].

MELERO, R. «El paisaje de los repositorios institucionales open access en España»[en línea].. BiD: textos universitaris de biblioteconomia $i$ documentació, 2008, n. 20. Disponible en: http://www.ub.edu/bid/20meler4.htm [consulta: 10 junio 2011].

MELERO, R. et al. Situación de los repositorios institucionales en España: informe 2009 [en línea].. Grupo de investigación Acceso Abierto a la Ciencia, 2009.

Disponible

en: http://digital.csic.es/bitstream/10261/11354/1/Informe2009-Repositorios_0.pdf [consulta: 10 junio 2011].

MENÉNDEZ SEOANE, S. "La planificación de bibliotecas públicas en España de 1978 a la actualidad" [en línea]. Revista general de información y documentación, 2007, v. 17, n. 2 p. 209-228. Disponible en: http://revistas.ucm.es/byd/11321873/articulos/RGID0707220209A.PDF [consulta: 9 Junio 2011].

MESA SÁNCHEZ, A. "Biblioteca verde. Experiencia UC3M" [en línea]. En: III Jornada profesional de la Red de bibliotecas del Instituto Cervantes. Madrid, 16 de diciembre de 2010. Disponible en: http://www.cervantes.es/ imagenes/File/biblioteca/jornadas/jornada_3/documentacion/mesa_sanchez_aur ora.pdf [consulta: 3 junio 2011].

MILLÁN, J.A. "El polimorfo libro electrónico". El profesional de la información, 2008, v. 14, n. 4, p. 369-371. 
MINNER VAN NEYGEN, V. "Sostenibilidad medioambiental y bibliotecas. Un nuevo grupo de interés para la IFLA" [en línea]. En: III Jornada profesional de la Red de bibliotecas del Instituto Cervantes. Madrid, 16 de diciembre de 2010. Disponible

en: http://www.cervantes.es/imagenes/File/biblioteca/jornadas/jornada_3/document acion/van neygen veerle.pdf [consulta: 3 junio 2011].

MINISTERIO DE CULTURA (a). Observatorio de la lectura y el Libro. [en línea] Disponible en: http://www.mcu.es/libro/CE/ObservatorioLecturaLibro.html 23/5/2011 [consulta: 3 junio 2011].

MINISTERIO DE CULTURA (b). El Ministerio de Cultura pone en marcha un servicio de préstamo de libros electrónicos en las Bibliotecas Públicas del Estado. [en línea]. 12 Enero 2011 Disponible en: http://www.mcu.es/gabineteprensa/ mostrarDetalleGabinetePrensaAction.do?prev_layout=notas\&layout=notas\&ca che=init\&html=24082011 nota.txt [consulta: 3 junio 2011].

MINISTERIO DE CULTURA (c). Situación actual y perspectivas del libro digital en España [en línea]. Madrid: Observatorio de la Lectura y el Libro, 2011. Disponible en: http://www.mcu.es/libro/docs/MC/CD/Ebook_2010.pdf [consulta: 3 junio 2011].

MÚNOZ DE SOLANO Y PALACIOS, B. (a) "Aspectos económicos del proceso de preservación digital" [en línea]. Revista española de Documentación Científica, 2006 , v. 29 , n. 4 , p. 572587 . http://redc.revistas.csic.es/index.php/redc/ article /view/309/384 [consulta: 3 junio 2011].

MÚNOZ DE SOLANO Y PALACIOS, B. (b) "El profesional de la información digital: Aspectos organizativos". Anales de Documentación, 2006, v. 9, p. 143163.

MUÑOZ MUÑOZ, A. M. y ARGENTE JIMÉNEZ, M. "Red de Centros de Documentación y Bibliotecas de Mujeres: cooperación entre las bibliotecas feministas españolas". El profesional de la información, 2010, v. 19, n. 5, p. 504509.

OMEYA CLAPAROLS, E.; PERMANYER BASTARDAS, J. y VILAGROSA ALQUÉZAR, E. "Instrumentos básicos para la planificación estratégica del servicio de biblioteca pública" [en línea]. BiD: textos universitaris de biblioteconomia $i$ documentació, 2009, núm. 23 Disponible en: http://www.ub.edu/bid/23/omella2.htm [consulta: 9 junio 2011].

ORERA ORERA, L. (ed.) La biblioteca universitaria. Madrid: Síntesis, 2005.

PEREZ ARRANZ, F. y MOSCOSO, P. "El libro electrónico y su incidencia en las bibliotecas universitarias y científicas españolas". Revista española de Documentación Científica, 2007, v. 30, n 3, p. 343-363.

PÉREZ IGLESIAS, J. "Bibliotecas en tiempos de crisis. ¿qué nos ofrecen y para qué las queremos?" Mi Biblioteca, 2009, n 17, p. 30-34.

PÉREZ-MONTORO GUTIÉRREZ, M. Gestión del conocimiento en las organizaciones: fundamentos, metodología y praxis. Gijón: Trea, 2008. 
PÉREZ PULIDO, M. "La enseñanza de la ética y deontología de la información en los estudios universitarios de biblioteconomía y documentación" [en línea]. BiD: textos universitaris de biblioteconomia i documentació, 2004, n. 13. Disponible en: http://www.ub.edu/bid/13pulid2.htm [Consulta: 10 junio 2011].

PÉREZ PULIDO, M. "Captar y generar recursos en tiempos de crisis: oportunidades de fundraising en bibliotecas públicas". En La biblioteca pública frente a la recesión: acción social y educativa. Murcia: Ediciones Tres Fronteras, ANABAD, 2010, p. 53-64.

PINTO, M.; BALAGUÉ, N. y ANGLADA, L. "Evaluación y calidad en las bibliotecas universitarias: experiencias españolas entre 1994-2006". Revista Española de Documentación Científica, 2007, v. 30, n. 3, p. 364-389.

PINTO, M; FERNÁNDEZ MARCIAL, V. y GÓMEZ-CAMARERO, G. "La herramienta «BiQual» como instrumento para el estudio de la calidad del servicio en bibliotecas universitarias españolas de ciencia y tecnología" [en línea]. Revista española de Documentación Cientifica, 2007, V. 30, N. 4, P. 465-491. Disponible en: http://redc.revistas.csic.es/index.php/redc/article/view/400/412 [consulta: 9 junio 2011].

REINA SEGOVIA, M. "Bibliotecas móviles y crisis económica. Una visión esperanzadora del valor social de nuestro trabajo" [en línea]. En IV Congreso Nacional de bibliotecas móviles. León, 2009. Disponible en: http://www.bibliobuses.com/documentos/Bibliobuses\%20y\%20crisis\%20econo mica.pdf [consulta: 3 junio 2011].

REINA SEGOVIA, M. "Los servicios bibliotecarios móviles y sus profesionales frente a la crisis económica. Encrucijadas y cambios". Educación y Biblioteca, 2010, n. 178, p. 93-96.

RIAZA CHAPARRO, M.I. "Cuando hay que hacer más con menos". Educación y Biblioteca, 2010, n. 178, p. 97-100.

RICO, I. y CALVO, C. "El proceso de evaluación EFQM como instrumento de mejora continua y reconocimiento en las bibliotecas" [en línea]. En II Jornadas Universitarias de Calidad y Bibliotecas, Málaga del 20 al 21 de mayo de 2010. Disponible en: http://dspace.uma.es/xmlui/bitstream/handle /10630/4192/taller_2.pdf?sequence $=1$ [consulta: 9 junio 2011].

RODRÍGUEZ BRAVO, B. «Los repositorios de información, guardianes de la memoria digital». Anales de Documentación, 2007, n. 10, p. 361-374.

RODRÍGUEZ BRAVO, B. y OLEA, I. "La rentabilidad de las revistas electrónicas. El caso de Emerald en la Universidad de León" [en línea]. En XII Jornadas de Gestión de la Información. Valor económico de la información: mercados, servicios y rentabilidad, Madrid, noviembre de 2010. Disponible en: http://eprints.rclis.org/bitstream/10760/12010/2/pres_rodriguez_olea.pdf [consulta: 10 junio 2011].

SALABERRIA, R. "La biblioteca en tiempos de crisis". Educación y Biblioteca, 2010, n. 178, p. 42-44. 
SAN JOSÉ MONTANO, B. La gestión de la colección en cooperación en las bibliotecas universitarias del siglo XXI. [en línea]. Madrid, Universidad Carlos III, 2011. http://e-archivo.uc3m.es/handle/10016/10412 [consulta: 3 junio 2011].

SÁNCHEZ GALÁN, J.I. Comunidades de práctica para el servicio de Biblioteca de la Universidad de Alcalá de Henares [en línea] BUAH, Documentos de trabajo, 2009. Disponible en: http:/dspace.uah.es/ jspui/bitstream/10017/2403/1/ comundp.pdf [consulta: 9 junio 2011].

SEDANO RUIZ, A.E. "La biblioteca verde: nuestro compromiso con la sostenibilidad y el medio ambiente. La experiencia de la biblioteca de la universidad de Burgos" [en línea]. En III Jornada profesional de la Red de bibliotecas del Instituto Cervantes. Madrid, 16 de diciembre de 2010. Disponible en: http://www.cervantes.es/imagenes/File/biblioteca/jornadas/jornada_3/ documentacion/ruiz_sedano_ana_esther.pdf [consulta: 3 junio 2011].

SEDIC. Jornada profesional "Là responsabilidad social corporativa en las organizaciones", Marzo 2011. Disponible en: http://www.sedic.es/actividadesjornada_RSC_30marzo2011.asp [consulta: 15 junio 2011].

Segunda Encuesta sobre el libro digital en España. Impacto de la digitalización en el catálogo, canales de distribución y de venta y política comercial. Marzo, 2011. Disponible en: http://www.federacioneditores.org/0_Resources/ Documentos/ SegundaEncuesta_LibroDigital2011.pdf [consulta: 15 junio 2011].

SUNYER, S. "Centros de recursos para el aprendizaje y la investigación -CRAI- y servicios bibliotecarios estratégicos para una Europa basada en el conocimiento" [en línea]. Intangible capital, 2006, n, 4, v. 2, p. 327-337. Disponible en: http://redalyc.uaemex.mx/pdf/549/54920401.pdf [consulta: 3 junio 2011].

TALADRIZ, M. "Estrategias bibliotecarias para el fomento del aprendizaje". Boletín de la Asociación Andaluza de Bibliotecarios, 2004, t. 54, n. 1-2, p. 273-280.

TEJADA-ARTIGAS, C., MENDO-CARMONA, C., RAMOS-SIMÓN, L., MARTÍNEZ COMECHE, J.A. y MOREIRO GONZÁLEZ, J.A. "Competencias y formación universitaria: la necesidad de un nuevo enfoque" [en línea]. En $8^{\circ}$ Congreso Nacional de Bibliotecología y Ciencias de la Información, Cartagena de Indias (Colombia), 31 May - 2 June 2006. Asociación Colombiana de Bibliotecólogos y Documentalistas - ASCOLBI. Disponible en: http://eprints.rclis.org/bitstream/10760/7930/1/Ascolbi

Congreso_2006_Ponencia_Carlos_Tejada.pdf [consulta: 3 junio 2011].

TÉRMENS GRAELLS, M. "Los consorcios, una nueva etapa de la cooperación bibliotecaria". El profesional de la información, 2005, v. 14, n. 3, p. 166-173.

TÉRMENS GRAELLS, M. "Investigación y desarrollo en preservación digital: un balance internacional". El profesional de la información, 2009, v. 18, n. 6, p. 613-624.

TORRES SALINAS, D. "Web 2.0 y biblioteca: de la experimentación a la evaluación" [en línea]. En: V Congreso Nacional de bibliotecas públicas. 
Gijón, 2010. Disponible en: http://www.mcu.es/bibliotecas/docs/MC/2010/ CongresoBP/ DanielTorres.pdf [consulta: 3 Junio 2011].

TORRES SANTODOMINGO, M. "La función social de las bibliotecas universitarias". Boletín de la Asociación Andaluza de Bibliotecarios, 2005, n. 80, p. 43-70.

VIVES I GRÀCIA, J. «Aspectos de propiedad intelectual en la creación y gestión de repositorios institucionales». El profesional de la información, 2005, v. 14, n. 4, p. 267278. 